Sociohistórica

ISSN: $1852-1606$

publicaciones@fahce.unlp.edu.ar

Universidad Nacional de La Plata

Argentina

\title{
Valorización dependiente y estancamiento en la Argentina a comienzos de siglo XXI
}

\author{
López, Emiliano; Barrera Insua, Facundo \\ Valorización dependiente y estancamiento en la Argentina a comienzos de siglo XX \\ Sociohistórica, núm. 46, 2020 \\ Universidad Nacional de La Plata, Argentina \\ DOI: https://doi.org/10.24215/18521606e108
}

Atribución no comercial compartir igual (CC BY-NC-SA) 4.0 
Artículos

\title{
Valorización dependiente y estancamiento en la Argentina a comienzos de siglo XXI
}

\author{
Dependent valorization and stagnation in Argentina at the beginning of the 21st century
}

Emiliano López

DOI: https://doi.org/10.24215/18521606e108

Facultad de Humanidades y Ciencias de la Educación-

Universidad Nacional de La Plata / Instituto de

Investigaciones en Humanidades y Ciencias Sociales-

CONICET, Argentina

elopez@fahce.unlp.edu.ar

Facundo Barrera Insua

Universidad Nacional de La Plata / Instituto de Desarrollo

Económico y Social, CONICET / Instituto de Ciencias

Sociales y Administración de la Universidad Nacional

Arturo Jauretche, Argentina

fbarrera@fahce.unlp.edu.ar

Recepción: 14 Junio 2019

Aprobación: 06 Febrero 2020

\section{ReSUMEN:}

A partir de la reinterpretación de las Cuentas Nacionales en términos de categorías marxianas, el presente texto tiene como objeto brindar una clave interpretativa para pensar el estancamiento de la economía argentina en relación con la dinámica de valorización dependiente del ciclo del capital internacional. Para ello, con información publicada en las Matrices Insumo-Producto (MIPINDEC, 1997 y 2004) y la construcción de series temporales (1997-2015), se estudia el desenvolvimiento de la economía en torno a los tres momentos del ciclo del capital (circulación inicial-producción-circulación final). Finalmente, del análisis empírico se concluye que, desde la necesidad de capital extranjero y maquinaria importada en el comienzo del ciclo, hasta la producción con destino en el exterior como componente principal de la demanda agregada, la economía argentina presenta un patrón de dependencia que puso un límite estructural al desenvolvimiento del producto a partir de $2008 \mathrm{y}$, especialmente, entre los años 2012-2015.

Palabras ClaVe: Ciclo del capital, Dependencia estructural, Estancamiento, Argentina.

\section{Abstract:}

Based on the reinterpretation of the National Accounts in terms of Marxian categories, this text aims to provide an interpretative key to think about the stagnation of the Argentine economy in relation to the dependent dynamics of capital valorization. For this, with information published in the Input-Output Matrices (MIP-INDEC, 1997 and 2004) and the construction of time series (1997-2015), we studied the development of the economy around the three moments of the capital cycle (initial circulationproduction-final circulation). Finally, the empirical analysis concludes that, from the need for foreign capital and machinery imported at the beginning of the cycle, to the production destined to the foreign market (as the main component of the demand), the Argentine economy has a dependency pattern that caused the stagnation of the product since 2008 and, especially, between the years 2012-2015.

KEYWORDS: Capital cycle, Structural dependency, Stagnation, Argentina.

\section{INTRODUCCIÓN}

La forma concreta que toma el proceso de acumulación de capital difiere significativamente entre países centrales y periféricos. El presente trabajo pretende poner en evidencia algunas de las características 
específicas de la dinámica de valorización del capital en una economía dependiente del ciclo del capital a escala global, como es el caso de la Argentina, que recurrentemente enfrenta períodos de stop and go.

En particular, estudiamos la tendencia al estancamiento de la economía argentina luego de la emergencia de un nuevo modo de desarrollo (López, 2015). A posteriori de la devaluación del peso, de la aceleración de la tasa de crecimiento y de la ganancia, emergieron tensiones en el proceso de valorización del capital que se hicieron evidentes hacia los años 2007-2008, y se profundizaron con la desaceleración de China, la caída del precio de los commodities y el impacto de la crisis global en Europa entre 2012 y 2015. Hacia finales de dicho período, tanto el crecimiento virtuoso del producto como de la tasa de acumulación de capital desaparecieron, y la economía argentina se encontró nuevamente en una fase de estancamiento de su ciclo económico.

A partir de la reinterpretación de las cuentas nacionales desde una perspectiva marxiana (Barrera y López, 2010; López y Barrera Insua, 2019), elaboramos series temporales desde el año 1997 para dar cuenta de las tensiones y los límites que implicó el proceso de acumulación y valorización del capital a escala nacional en los años 2002-2015. En la primera sección, presentamos el enfoque teórico-metodológico, las premisas para reinterpretar las estadísticas oficiales. En la segunda sección, aplicamos el esquema analítico a la caracterización de la Argentina como una economía dependiente durante el transcurso de los años 2002-2015. Allí incluimos los hallazgos empíricos, con especial énfasis en el estancamiento que se produjo entre 2012 y 2015. Por último, reflexionamos sobre las tensiones acumuladas en el patrón de acumulación vigente que son, a nuestro entender, producto de la dependencia estructural de la Argentina del ciclo del capital global.

\section{UN ESQUEMA TEÓRICO-METODOLÓgico PARA EL ESTUDiO DE LA VALORIZACIÓN DEL CAPITAL}

El capital entendido como relación social puede ser descrito como un ciclo (D-M-...-P-...M'-D') que comienza con un adelanto de capital en su forma monetaria (D) para comprar mercancías (M) utilizadas en el proceso productivo (M-...-P-.... $\mathrm{M}^{\prime}$ ) y que finaliza con una fase de circulación final $\left(\mathrm{M}^{\prime}-\mathrm{D}^{\prime}\right)$ en la cual las nuevas mercancías producidas son vendidas. El objetivo del ciclo completo es la valorización del capital en sí mismo $\left(\mathrm{D}^{\prime}>\mathrm{D}\right)$. La distribución del producto social, la dinámica del empleo de la fuerza de trabajo y aun las relaciones entre seres humanos y la naturaleza tienden a estar dominadas por la necesidad de obtención de plusvalor o excedente económico a ser apropiado de manera privada (Marx, 2007).

Es por eso que consideramos relevante estudiar el ciclo de acumulación de capital en la Argentina como la relación entre las esferas de la producción, distribución y circulación del producto social. Para ello utilizaremos algunas de las categorías más relevantes del análisis de Marx en El Capital, con objeto de analizar el proceso por el cual el ciclo que describimos es producido y reproducido, para luego adentrarnos en las particularidades del ciclo en la Argentina.

\section{1. Las categorías analíticas para el estudio del ciclo del capital}

Es sabido que, en el inicio de un ciclo de reproducción ampliada, el gasto de capital en su forma dinero se direcciona a materias primas, medios de producción y fuerza de trabajo. Por medio del proceso productivo estas mercancías son incluidas en la lógica del capital, lo que permite la generación de un Valor Total (VT) que está formado por el capital variable (V), el capital constante circulante (CC) y el plusvalor (P).

El proceso de trabajo no produce solamente los bienes necesarios para cubrir los costos de producción, sino que además genera un nuevo valor que se distribuye entre los pagos a la fuerza de trabajo y el plusvalor. Esto significa que, en buena medida, en la esfera de la producción se desarrolla la valorización del capital, es decir, la generación de P. Tal como planteaba Marx, "El misterio de la virtud de capital para valorizarse a si mismo 
es el poder para controlar un cierto monto de trabajo ajeno no remunerado" (Marx, 2006b, p. 649). Es decir, el capital alcanza la valorización si puede subordinar la producción de valor a su propia lógica, que es la lógica de la ganancia. Esto implica una forma particular de producción de bienes y servicios.

El tercer momento del ciclo se caracteriza por una nueva fase de circulación, asociada con la venta del valor generado en el proceso productivo (expresado en mercancías) e intercambiado por dinero. Es decir, el valor total generado en el proceso productivo (VT) se expresa por el lado de los ingresos. Sin embargo, según Shaikh y Tonak (1994) la expresión materializada del valor en mercancías es presentada como bienes y servicios, los cuales serán llamados producto total (PT), cuyas magnitudes deben igualar al valor total. La equidad VT-PT otorga un marco de consistencia de las diferentes esferas, es decir, entre la producción y la circulación de las mercancías en nuestras sociedades, que tienen a la relación capital como forma económica dominante.

Es necesario observar que, en el esquema desarrollado por Marx, hay una consistencia entre la fase inicial de circulación (D-M), la fase de producción (M-...-P-...-M $\mathrm{M}^{\prime}$ ) y la fase de circulación final $\left(\mathrm{M}^{\prime}-\mathrm{D}^{\prime}\right)$. Dada la consistencia entre los productos del trabajo y el ingreso generado en el proceso productivo, podemos estudiar los efectos co-determinantes de cada fase del ciclo sobre las otras. Respecto de la fase inicial, una de las categorías relevantes a medir empíricamente es la ratio de la fuerza de trabajo viva o empleo (L) y el trabajo muerto o capital fijo (C) incorporado en la producción, lo cual puede ser definido como la composición orgánica del capital (COC):

$$
\mathrm{COC}=\frac{C}{L}
$$

La COC no es más que la manifestación de la ratio capital-trabajo en la economía ortodoxa. Por ello, puede estimarse como una ratio de capital constante en términos reales y la masa de fuerza de trabajo aplicada a la producción (Saad-Filho, 1993). Este indicador da cuenta de la intensificación del cambio técnico que está detrás de la tendencia a la crisis en estas economías (Marx, 2006a).

Las proporciones de trabajo vivo y medios de producción que son utilizadas en el proceso productivo y aplicadas a las materias primas resultan en el valor total que incluye el nuevo valor o valor agregado. Podemos evaluar este proceso por medio del indicador llamado tasa de plusvalor (TPV), definido como la proporción de plusvalor $(S)$ en relación con la remuneración recibida por los trabajadores que venden su capacidad de trabajo o capital variable $(\mathrm{V})$ :

$$
\operatorname{TPV}=\bar{V}
$$

El indicador TPV indica la forma en la cual el ingreso es distribuido, primariamente, entre los productores directos y el plusvalor generado. Esta categoría es relevante para analizar el éxito del proceso de valorización del capital (PaitaridisyTsoulfidis, 2012).

Una cierta COC, por su parte, establece una productividad de la fuerza de trabajo (PFT), la cual puede definirse como la ratio entre el producto total y el empleo de la fuerza de trabajo:

$$
\mathrm{PFT}=\frac{P T}{L}
$$

Este indicador también implica una relación entre la fase inicial (D-M) y la fase de producción (M-...-P-...$\mathrm{M}^{\prime}$ ): el efecto que la composición del capital tiene sobre la cantidad física de bienes y servicios producidos y la forma de apropiación del plusvalor. La evolución relativa de la productividad y del ingreso de los trabajadores da cuenta de las estrategias de extracción de plusvalor seguidas por el capital (Lebowitz, 2005). 
Finalmente, es necesario discutir la realización del valor generado en la producción y la capacidad de acumulación en una escala nacional. Esto es, deberíamos observar algunas variables relevantes de la esfera de la circulación de la tercera fase del ciclo. En este sentido, es útil evaluar la tasa de ganancia de la economía en su conjunto $(\pi)$ o tasa de beneficios. Siguiendo los desarrollos de Marx (2006a), podríamos definirla como la ratio entre el plusvalor y el capital total adelantado, tanto el constante como el variable. Matemáticamente, la tasa de ganancia se expresa como:

$$
\pi=\frac{S}{C+V}
$$

Además, la capacidad de acumulación de una economía capitalista está asociada a las proporciones de producto final (PF) que se destinan a incrementar la asignación de capital fijo (I), al consumo suntuario $\left(\mathrm{CON}_{\mathrm{k}}\right)$ y a las exportaciones netas (X-IMPO). También incluimos como indicadores de esta tercera fase del ciclo del capital la participación del consumo de los trabajadores sobre el producto final $\left(\mathrm{CON}_{\mathrm{w}}\right)$ y el gasto público $(\mathrm{G})$.

En resumen, los indicadores que utilizamos son:

$$
\begin{aligned}
& \frac{I}{P F} \quad(5), \\
& \frac{C O N_{k}}{P F} \quad(6), \\
& \frac{X-I M P O}{P F}(7), \\
& \frac{C O N_{w}}{P F} \text { (8), } \\
& \frac{G}{P F} \quad \text { (9). }
\end{aligned}
$$

Estas categorías representan los "usos" del producto que ha sido generado en el ciclo completo. La tendencia al crecimiento y la expansión de las economías capitalistas requiere de la inversión en capital fijo para incrementar la productividad del trabajo. Aquellas en las que el consumo productivo como parte de la producción total es más elevado, poseerán mayores potencialidades de acumulación de capital y, por tanto, incrementarán las posibilidades de valorización futura. En contraste, altas proporciones de consumo suntuario y transferencias de valor al exterior -vía exportaciones netas- pueden resultar en una dinámica de acumulación poco virtuosa y decantar en un proceso de estancamiento, lo cual ha caracterizado a las economías latinoamericanas (Braun y Joy, 1981; Marini, 2007; Osorio, 2004).

Las variables que hemos propuesto serán de gran utilidad para evaluar el patrón particular de acumulación y valorización en la Argentina en la etapa 2002-2015. Al mismo tiempo, estas categorías serán útiles para responder la pregunta acerca de cómo las condiciones de economía dependiente y periférica de nuestro país impusieron límites al éxito de los procesos de crecimiento y acumulación de capital luego de 2008 y, sobre todo, entre 2012 y 2015.

Vale decir que el presente trabajo se inserta dentro de una variedad de estudios empíricos que han utilizado metodologías similares para estudiar las economías centrales: Shaikh yTonak(1994), Moseley(1997), Wolf (2001), Paitaridis y Tsoulfidis (2012) para Estados Unidos y Cockshott, Cottrell, y Michaelson (1995) para Gran Bretaña, entre otros. Sin embargo, dicha propuesta aparece con menor frecuencia en estudios para 
economías periféricas o semi-periféricas. Para estas últimas hemos podido contar con Maniatis (2005) para Grecia, Venida (2007) para Filipinas y Memis (2007) para Turquía.

\section{I.II La reinterpretación de las Cuentas Nacionales de la Argentina basada en las categorías marxianas}

El punto de partida para reconciliar las categorías de análisis usadas en el nivel abstracto con las fuentes empíricas es una re-interpretación de las Cuentas Nacionales mediante las matrices insumo-producto, que cuentan con una gran cantidad de información sobre la producción/reproducción de la riqueza de un país. Para el caso de la Argentina, la Matriz Insumo-Producto (MIP) es publicada por el Instituto Nacional de Estadística y Censos (INDEC), y cuenta con información de la estructura de ingresos y gastos por sector que se refieren al año 1997, y, por medio de su actualización, al año 2004. ${ }^{1}$ Así, permite una cuantificación del crecimiento del ingreso generado en cada sector de la economía -lado de los ingresos- y de la manera en la cual estos ingresos son gastados -lado de los usos-.

La MIP organiza las transacciones económicas entre diferentes actividades económicas y a su interior, es decir inter e intra-rama. Esta información da cuenta de la interrelación entre varios sectores y los impactos directos e indirectos que ellos tienen sobre la demanda final. Además, las tablas insumo-producto son una importante herramienta para la investigación empírica, dado que proveen un marco de consistencia para las estimaciones provenientes de diferentes fuentes (encuestas industriales, encuesta de gasto de los hogares, estadísticas de comercio exterior, entre otras) y tienen la ventaja de incluir datos que permiten calcular el capital circulante (CC). Más aún: de acuerdo con los criterios ortodoxos, las tablas dividen el valor agregado generado en cada sector entre los ingresos de capitalistas y trabajadores en un período.

Los datos concernientes a empleo y distribución del valor agregado entre asalariados y no asalariados en 2004 fueron obtenidos a través de la Cuenta de Generación de Ingreso, publicada por la Dirección de Cuentas Nacionales para el período 1993-2007, y compatible con los valores que surgen de las tablas de insumoproducto.

La reinterpretación de las tablas de insumo-producto se realizó a partir de la aplicación empírica de Shaikh y Tonak (1994) para la economía de Estados Unidos, atendiendo a la clasificación de trabajo productivoimproductivo. ${ }^{2}$ Sin embargo, a diferencia del mencionado texto, nuestra selección de actividades económicas involucra todas aquellas con un proceso de trabajo generador de plusvalor y que, a su vez, generan valores de uso pertenecientes a la esfera de la producción. Al mismo tiempo, incluimos aquellas actividades relacionadas con la realización -ventas mayoristas y minoristas- y redistribución del valor originado en la producción finanzas, alquileres, etc. -- como parte de la esfera de la circulación. El valor generado en la producción es redistribuido en los pagos de renta de la tierra, intereses y salarios, etc.

En las cuentas nacionales las actividades de circulación y el sector no capitalista son considerados como parte de las actividades productivas, mientras que para el enfoque marxiano no hay nueva generación de ingreso en las actividades secundarias. Estos flujos son considerados parte de las transacciones totales, pero no suman al valor total producido en la economía. De esta manera, el VT en términos marxianos siempre será menor que el que surge de las tablas de insumo-producto. Además, en el primer caso el valor agregado es obtenido por la deducción del capital constante circulante del valor total; por tanto, es también menor que en las cuentas nacionales, en las que, para la obtención del valor agregado, se resta el total de las compras intermedias. El capital variable, por su parte, será equivalente a la masa salarial de los trabajadores en la esfera de la producción.

Por otro lado, el plusvalor se obtiene de la diferencia entre el valor agregado y la masa salarial. Este concepto se corresponde con el excedente bruto de explotación que calculan las cuentas oficiales. El plusvalor será más elevado que el excedente bruto de explotación, debido a que el excedente bruto de explotación en las cuentas 
nacionales se calcula tomando en cuenta el promedio de salarios del conjunto de la economía, mientras que aquí consideramos la masa salarial del sector productivo.

Si miramos el lado de los usos, podemos encontrar diferencias en el tercer momento del ciclo del capital. En el formato de cuentas ortodoxas, el producto total es la suma de los inputs intermedios y el producto final, para todas las esferas -circulación, producción y sector no capitalista-, mientras que el enfoque marxiano sólo incluye las actividades productivas. De esta manera, el producto total en la perspectiva marxiana será menor que el que se refleja en las cuentas oficiales. ${ }^{3}$

Así, bajo la clasificación marxiana, las ramas de actividad de las tablas insumo-producto pueden reinterpretarse en función de las esferas de producción, circulación y sectores no capitalistas (Tabla1).

TABLA 1

Clasificación marxiana de las actividades económicas de las tablas insumo-producto

\begin{tabular}{|c|c|}
\hline $\begin{array}{l}\text { Clasificación } \\
\text { marxiana }\end{array}$ & $\begin{array}{l}\text { Actividades económicas en las tablas insumo- } \\
\text { producto. Argentina }\end{array}$ \\
\hline $\begin{array}{l}\text { Esfera de la } \\
\text { Producción }\end{array}$ & $\begin{array}{l}\text { Agricultura, Ganadería, Caza, Sivicultura y Pesca } \\
\text { Minería } \\
\text { Industrias Manufactureras } \\
\text { Construcción } \\
\text { Suministros de Electricidad, Gas y Agua } \\
\text { Transporte y Almacenamiento }\end{array}$ \\
\hline $\begin{array}{l}\text { Esferas de la } \\
\text { circulación }\end{array}$ & $\begin{array}{l}\text { Comercio } \\
\text { Hoteles y Restaurantes } \\
\text { Correos y Telecomunicaciones } \\
\text { Enseñanza y Salud } \\
\text { Intermediación Financiera } \\
\text { Act. Inmoviliarias, Empresariales y de Alquiler }\end{array}$ \\
\hline $\begin{array}{l}\text { Sector No } \\
\text { Capitalista }\end{array}$ & $\begin{array}{l}\text { Administración Pública y Defensa } \\
\text { Act. Comunitarias, Sociales y Personales } \\
\text { Servicio Doméstico }\end{array}$ \\
\hline
\end{tabular}

Fuente: Elaboración propia sobre la base de datos de MIP-INDEC

\section{I.III La construcción de series temporales}

Las tablas de insumo-producto permiten construir los valores de partida para aquellas variables que no tienen periodicidad anual en las publicaciones de Cuentas Nacionales de Argentina, en particular, los datos correspondientes al consumo de bienes intermedios (o capital constante circulante). En este sentido, el punto de inicio para el cálculo de las series temporales anuales es de Valor Bruto de Producción -o Valor Total-, que requiere, en primer lugar, de los valores iniciales del capital circulante obtenidos de la MIP, equivalente a la suma de las compras intermedias de cada sector. Sobre la base de estos valores iniciales, realizamos luego una interpolación lineal similar a la que desarrollan Shaikh y Tonak (1994) en su trabajo sobre Estados Unidos. Esta interpolación se basa en la fórmula:

$$
C C^{t}=C C^{0}+\frac{C C^{1}-C C^{0}}{\left(V A^{1}-V A^{0}\right)\left(V A^{t}-V A^{0}\right)}
$$


donde, $\mathrm{CC}^{0}, \mathrm{CC}^{1}$ y $\mathrm{CC}^{\mathrm{t}}$ representan las proporciones de capital circulante sobre valor agregado para los años base (1997), último dato (2015) y el año que se intenta calcular (entre 1998 y 2014) que extrajimos en los dos primeros casos de las matrices insumo producto y de las tablas de utilización que comenzó a publicar el INDEC en 2016. Por su parte, $\mathrm{VA}^{0}, \mathrm{VA}^{1} \mathrm{y} \mathrm{VA}^{\mathrm{t}}$ representan los valores agregados para los años base (1997), último dato (2015) y el año corriente (valor que se pretende estimar), respectivamente. Este cálculo nos otorga un valor para el cociente de capital circulante y valor agregado para el año corriente, que luego multiplicamos por el valor agregado corriente para obtener el dato de capital circulante en millones de pesos para cada año.

Con esta serie de capital circulante, logramos calcular el VT y desde allí realizar un cálculo para cada una de las categorías mencionadas en las ecuaciones de (1) a (9).

Finalmente, las series temporales de categorías marxianas para el período 1997-2015 las presentamos en la Tabla $2 .{ }^{4}$

TABLA 2

Estimación de las categorías marxianas en la Argentina. Período 1997-2015. En cocientes y porcentajes

\begin{tabular}{|c|c|c|c|c|c|c|c|c|c|c|c|c|c|c|c|c|c|c|c|}
\hline Indicadores & 1997 & 1998 & 1999 & 2000 & 2001 & 2002 & 2003 & 2004 & 2005 & 2006 & 2007 & 2008 & 2009 & 2010 & 2011 & 2012 & 2013 & 2014 & 2015 \\
\hline $\mathrm{PF}$ & $9,4 \%$ & $2,6 \%$ & $-5,3 \%$ & $-1,0 \%$ & $-6,7 \%$ & $-13,2 \%$ & $17,3 \%$ & $15,3 \%$ & $8,8 \%$ & $13,5 \%$ & $16,8 \%$ & $17,7 \%$ & $0,7 \%$ & $15,2 \%$ & $17,9 \%$ & $-5,1 \%$ & $-0,1 \%$ & $-0,5 \%$ & $-2,8 \%$ \\
\hline $\mathrm{COC}$ & 56,0 & 57,6 & 56,7 & 54,7 & 53,4 & 66,6 & 67,1 & 67,2 & 65,3 & 67,0 & 63,2 & 59,1 & 59,3 & 53,3 & 53,6 & 48,1 & 44,3 & 39,8 & 35,5 \\
\hline TPV & 1,7 & 1,6 & 1,5 & 1,5 & 1,4 & 2,0 & 2,0 & 1,9 & 1,7 & 1,5 & 1,5 & 1,4 & 1,2 & 1,3 & 1,2 & 1,1 & 1,1 & 1,5 & 1,6 \\
\hline PFT & 51,5 & 51,1 & 49,5 & 50,7 & 50,4 & 45,9 & 48,8 & 49,6 & 50,8 & 54,0 & 59,3 & 66,8 & 69,7 & 69,9 & 76,4 & 72 & 71 & 75 & 72 \\
\hline$\pi$ & $18,9 \%$ & $18,1 \%$ & $17,0 \%$ & $17,6 \%$ & $17,3 \%$ & $15,1 \%$ & $15,8 \%$ & $16,6 \%$ & $16,9 \%$ & $16,5 \%$ & $18,6 \%$ & $20,6 \%$ & $19,0 \%$ & $23,2 \%$ & $25,4 \%$ & $25,2 \%$ & $26,7 \%$ & $35,2 \%$ & $37,4 \%$ \\
\hline $\mathrm{CONW/FP}$ & $29,8 \%$ & $30,6 \%$ & $32,6 \%$ & $32,9 \%$ & $34,6 \%$ & $27,1 \%$ & $25,9 \%$ & $26,1 \%$ & $26,9 \%$ & $28,4 \%$ & $28,5 \%$ & $29,7 \%$ & $32,8 \%$ & $32,7 \%$ & $34,3 \%$ & $37,6 \%$ & $39,1 \%$ & $34,1 \%$ & $33,6 \%$ \\
\hline CONk/FP & $32,5 \%$ & $31,1 \%$ & $30,1 \%$ & $30,0 \%$ & $28,7 \%$ & $27,0 \%$ & $28,7 \%$ & $26,8 \%$ & $24,9 \%$ & $22,3 \%$ & $21,9 \%$ & $31,1 \%$ & $20,0 \%$ & $19,7 \%$ & $17,0 \%$ & $15,5 \%$ & $14,3 \%$ & $18,4 \%$ & $20,8 \%$ \\
\hline I/FP & $17,4 \%$ & $17,8 \%$ & $16,1 \%$ & $14,7 \%$ & $13,0 \%$ & $10,4 \%$ & $13,1 \%$ & $16,1 \%$ & $17,6 \%$ & $19,0 \%$ & $19,5 \%$ & $18,9 \%$ & $17,0 \%$ & $17,5 \%$ & $17,8 \%$ & $16,7 \%$ & $16,7 \%$ & $16,9 \%$ & $16,9 \%$ \\
\hline G/FP & $10,8 \%$ & $11,2 \%$ & $12,3 \%$ & $12,5 \%$ & $13,0 \%$ & $10,7 \%$ & $9,9 \%$ & $9,4 \%$ & $9,8 \%$ & $10,0 \%$ & $10,4 \%$ & $10,9 \%$ & $12,9 \%$ & $12,6 \%$ & $13,4 \%$ & $14,6 \%$ & $15,2 \%$ & $15,5 \%$ & $17,0 \%$ \\
\hline$X / F P$ & $9,5 \%$ & $9,3 \%$ & $8,8 \%$ & $10,0 \%$ & $10,6 \%$ & $24,8 \%$ & $22,4 \%$ & $21,6 \%$ & $20,8 \%$ & $20,3 \%$ & $19,8 \%$ & $19,5 \%$ & $17,3 \%$ & $17,5 \%$ & $17,5 \%$ & $15,6 \%$ & $14,6 \%$ & $15,1 \%$ & $11,6 \%$ \\
\hline$X-I M P O / F P$ & $-2,0 \%$ & $-2,3 \%$ & $-1,5 \%$ & $-0,6 \%$ & $1,2 \%$ & $13,1 \%$ & $9,7 \%$ & $6,2 \%$ & $5,0 \%$ & $4,6 \%$ & $3,5 \%$ & $3,0 \%$ & $4,3 \%$ & $2,4 \%$ & $1,4 \%$ & $1,7 \%$ & $-0,2 \%$ & $0,2 \%$ & $-1,4 \%$ \\
\hline
\end{tabular}

II. DEL BOOM AL ESTANCAMIENTO: LA DEPENDENCIA COMO UN LÍMITE ESTRUCTURAL PARA LA ACUMULACIÓN

La caracterización de la Argentina como una economía dependiente parte de abordar el proceso que llevó a la economía del boom al estancamiento durante el transcurso de los años 2002-2015. A nuestro entender, el hecho central que marca la tendencia al estancamiento en Argentina se vincula con los patrones de dependencia de nuestro país.

Durante la etapa de circulación inicial (D-M), dos aspectos definen el carácter periférico de nuestra economía: el primero de ellos es que el dinero utilizado para iniciar el ciclo es mayormente extranjero; la segunda cuestión es que la mayor parte de este dinero se destina a la compra de mercancías importadas, en especial insumos y maquinaria (Marini, 2007; Barrera y López, 2010). Estas condiciones, a su vez, se expresan en la esfera de la circulación final, dado que la alta proporción de capital transnacional al inicio del ciclo restringe el uso del excedente a escala doméstica a través de la remisión de utilidades. De esta manera, el excedente se produce en la periferia y se transfiere hacia el centro. Asimismo, cuando la economía crece sin desarrollar íntegramente la estructura productiva se incrementan también los requerimientos de dólares para solventar los gastos de importación (Braun y Joy, 1981; Gigliani y Michelena, 2013).

Durante la fase de producción (M-P-M`), a partir de la integración de los países periféricos en los mercados globales con un rol predominante de los capitales extranjeros, aparecen en la economía local diferenciales en la productividad del trabajo entre ramas con indicadores de concentración del capital más altos y más bajos: la productividad es más alta allí donde se ubican los capitales transnacionales, en relación con otras ramas con capitales pequeños y medianos (Marini, 2007). 
Estas particularidades de las economías periféricas llevan a una mayor desigualdad entre ramas de actividad (y en su interior) y una tasa de plusvalor más elevada en comparación con las economías centrales, producto de una tendencia de los capitales pequeños y medianos de recurrir a fuentes de extracción de plusvalor absoluto (mayores niveles de precarización del trabajo, entre otras posibilidades), que permitan morigerar sus pérdidas en la distribución del excedente (Marini, 2007; Barrera y López, 2010). ${ }^{5}$

Finalmente, el patrón de demanda final $\left(\mathrm{M}^{\prime}-\mathrm{D}^{\prime}\right)$ en la periferia implica la consideración de dos factores. Primero, dado que el mercado doméstico es reducido, la exportación de productos es uno de los componentes centrales de la demanda. Segundo, el hecho de que el ingreso se encuentre más concentrado en las manos de los empresarios y las clases dominantes hace que el consumo de lujo sea un porcentaje importante del consumo total (Marini, 2007).

A partir de esta lectura rápida de los factores que impactan en la posición dependiente de América Latina en general, y de la Argentina en particular, nos preguntamos si es posible pensar que estos condicionamientos estructurales puedan encontrarse en la base de la tendencia al estancamiento de los años posteriores a 2008, que actuaron, en definitiva, como una barrera a la continuidad del modo de desarrollo post-neoliberal.

Sobre la base de la información estadística que sintetizamos en la Tabla 2, podemos distinguir dos tipos de tensiones sobre la economía argentina que se desarrollaron a lo largo del período 2008-2015: las internas y las externas. Entre las tensiones internas, encontramos al menos cuatro aspectos a desarrollar: los conflictos distributivos, el estancamiento de la productividad del trabajo, la baja tasa de inversión y la estructura productiva desequilibrada. Por su parte, las tensiones externas se vincularon con los limitantes comerciales, la remisión de utilidades y la fuga de capitales.

$\mathrm{Al}$ rastrear dichas tensiones es posible diferenciar tres sub-períodos en los años 2002-2015. En primer lugar, el período de emergencia y estabilización de un nuevo modelo de desarrollo (2002-2007). En segundo lugar, la aparición de tensiones en el nuevo modelo (2008-2011), que comenzaron a desarrollarse desde la llamada "crisis del campo", con impactos tanto en el plano económico (Schorr, 2018), como el político (López y Cantamutto, 2018). Por último, una etapa en la cual las tensiones acumuladas durante esos años se intensifican y conducen a una situación de estancamiento del patrón de acumulación que, además, dio lugar a una recomposición política de los sectores más concentrados del empresariado, que lograron acordar una propuesta político-partidaria para las elecciones de 2015.

En la primera etapa, que se inicia con la caída de la convertibilidad del peso en 2002 y una devaluación del orden del $400 \%$, los indicadores del proceso de acumulación experimentaron una dinámica de crecimiento virtuosa. En el curso de cinco años el producto final registró un incremento acumulado del $76 \%$. Del mismo modo, la tasa de ganancia trepó un $33 \%$ entre 2002 y 2008 (Ver figura 1). 
FIGURA 1

Variación del producto final y de tasa de ganancia (eje secundario) para la Argentina en términos porcentuales. Período 1997-2015

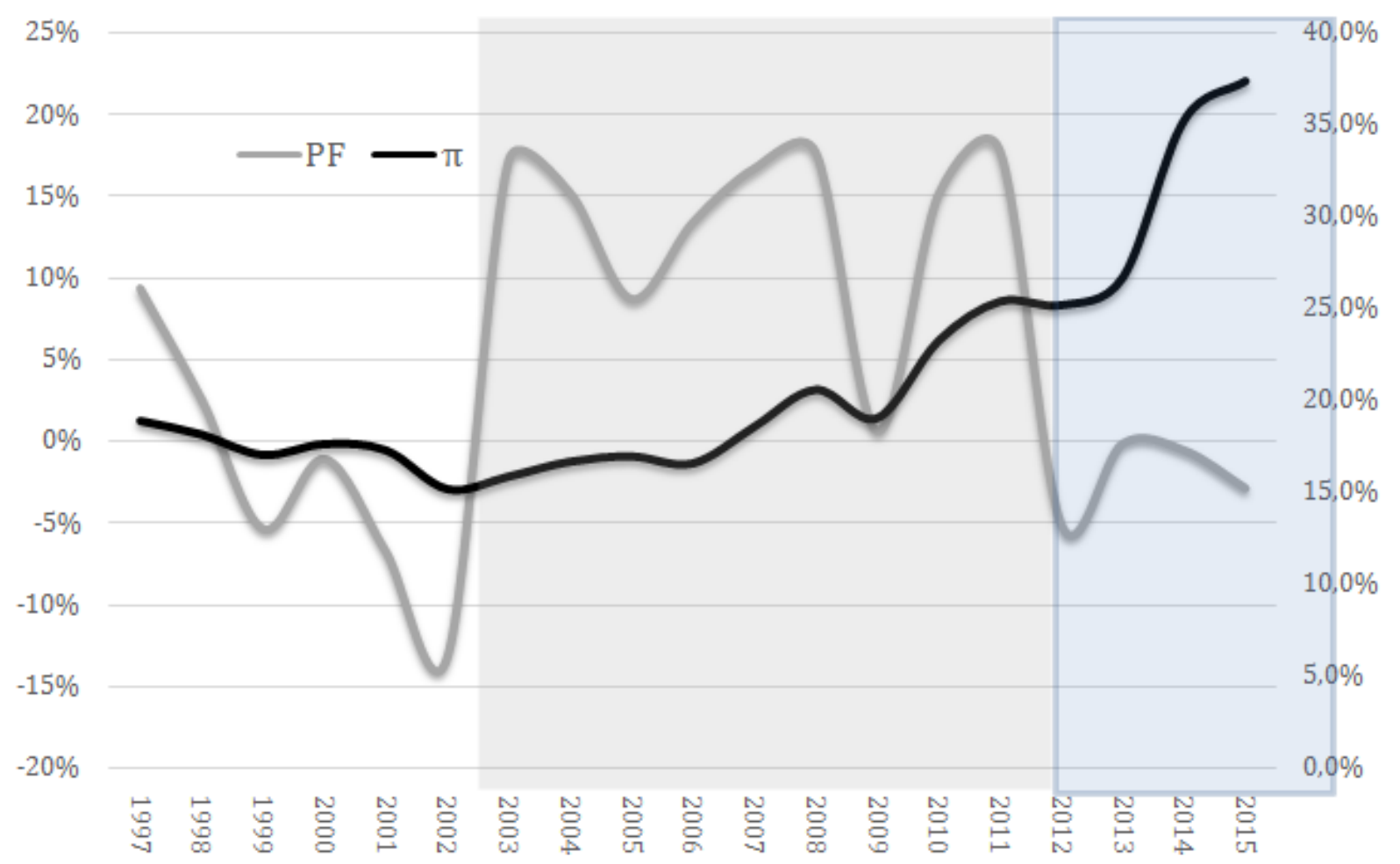

Fuente:Elaboración propia sobre la base de datos del Ministerio de Hacienda

Estas variables dan cuenta de que el boom de la economía tiene como contracara la caída de la COC y el incremento de PFT, y una nueva estructura del producto final, esencialmente realizado en la exportación, cuestiones que en conjunto marcan la resolución de la crisis del proyecto neoliberal. Así, la economía experimentó varios años de altas tasas de crecimiento y altos niveles de reservas en el Banco Central que posibilitaron una reducción importante del endeudamiento externo. 


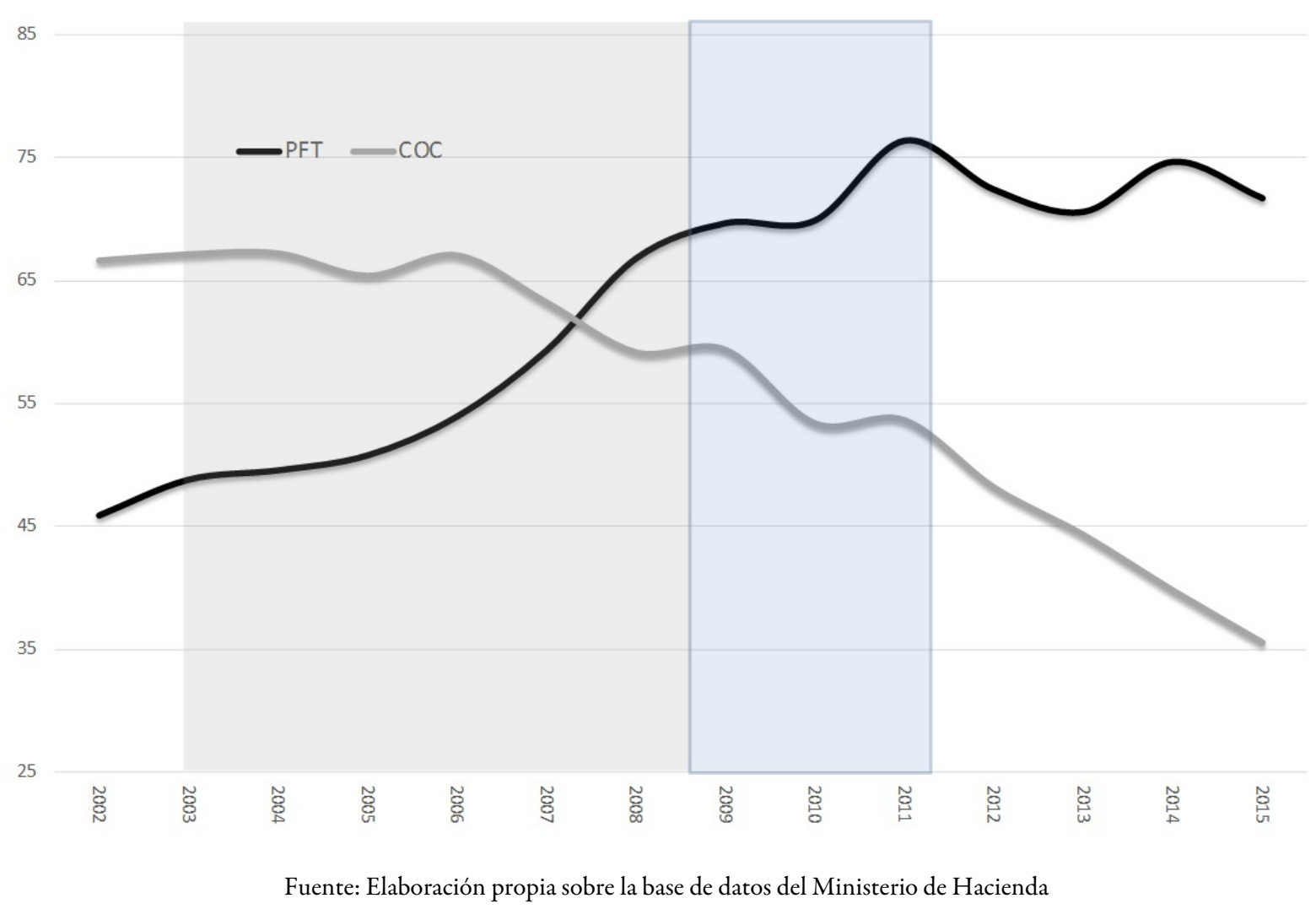

Sin embargo, como marcamos en las Figuras 1 y 2, en 2008 se produjo un quiebre en este proceso virtuoso de acumulación. Vale destacar que cuando la crisis financiera global estalló, la situación macroeconómica de América Latina era sólida, y la Argentina no fue la excepción. Por ello, el impacto de la caída de los precios de los commodities y la reversión del flujo de capitales fue limitado. ${ }^{6}$ Aun así, en ese momento las tensiones comenzaron a emerger. Veamos cada una de ellas de manera detallada.

En primer lugar, veamos la tensión distributiva. En los comienzos del nuevo modo de desarrollo, la redistribución de ingresos a favor de las clases dominantes \#que siguió a la devaluación del peso en un contexto de desempleo masivo y altos niveles de pobreza\#, permitió que se desarrollase el proceso de acumulación de manera exitosa. Es decir, el impulso inicial que permitió el crecimiento del producto y las ganancias fue el resultado de una redistribución de ingresos regresiva. Sin embargo, las demandas de los sindicatos y su fortaleza en la negociación durante el período redujeron sensiblemente la explotación de los trabajadores, lo que llevó a una tensión cada vez de mayor en la distribución del excedente (Barrera Insua, 2015). Esto se ve representado en la caída de más de $30 \%$ entre 2002 y 2012 de la tasa de plusvalor, que representa, como hemos dicho, la relación distributiva clave entre trabajo y capital. 
FIGURA 3

Evolución de la tasa de plusvalor para la Argentina. Período 1997-2015

80

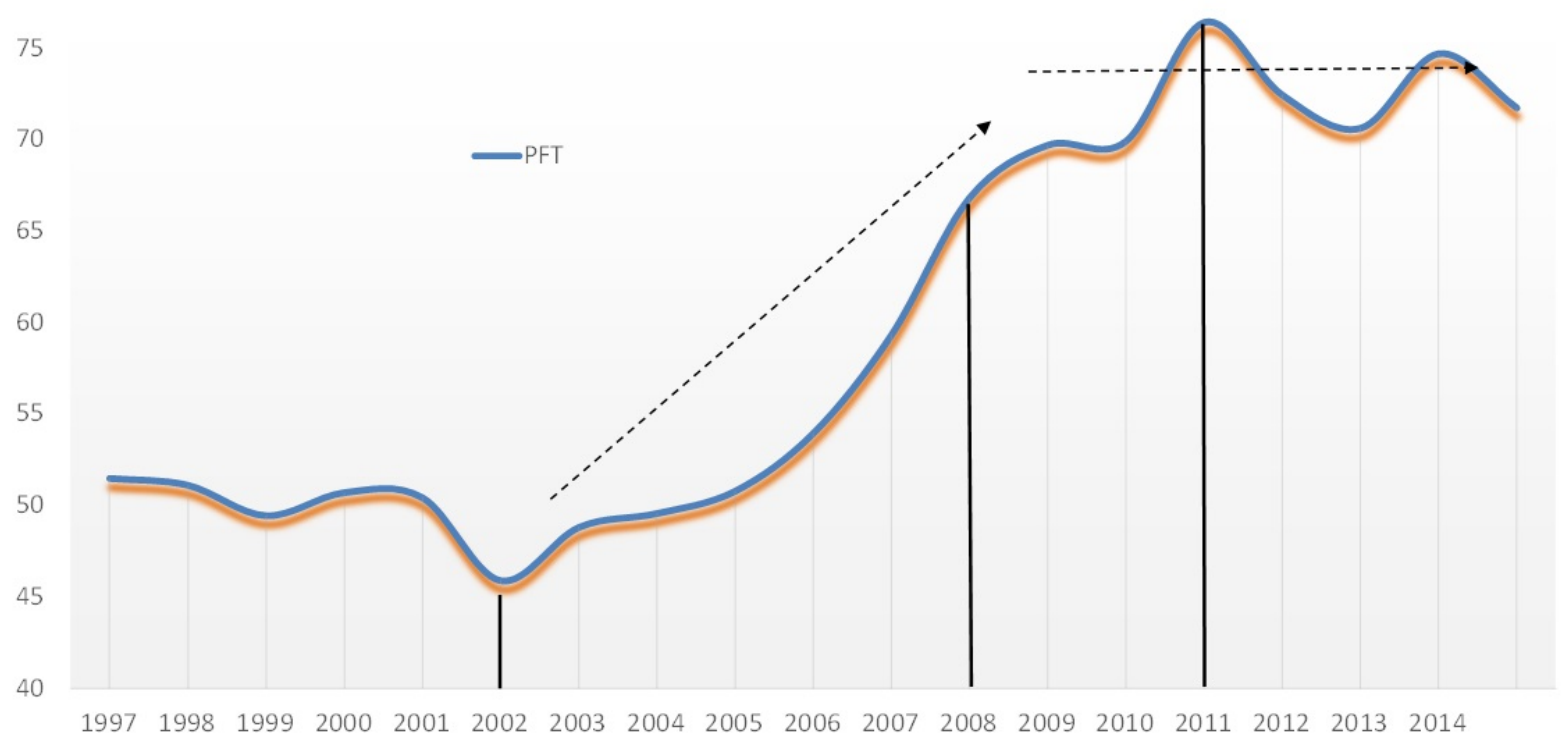

Fuente: Elaboración propia sobre la base de datos de Ministerio de Hacienda y Ministerio de Trabajo

Esta tendencia tiene su momento final en 2013 y se revierte con devaluaciones superiores al $30 \%$ durante dicho año y el siguiente, con lo cual la tasa de ganancia se incrementa a causa de una nueva redistribución regresiva.

En segundo lugar, el cambio en la pendiente de la curva de PFT a partir de 2008 y la caída desde 2011 reforzaron la tendencia al conflicto distributivo, dadas las menores posibilidades de las clases dominantes para apropiarse de una porción creciente de valor (Figura 4).

FIGURA 4

Productividad media del trabajo para la Argentina. Período 1997-2015

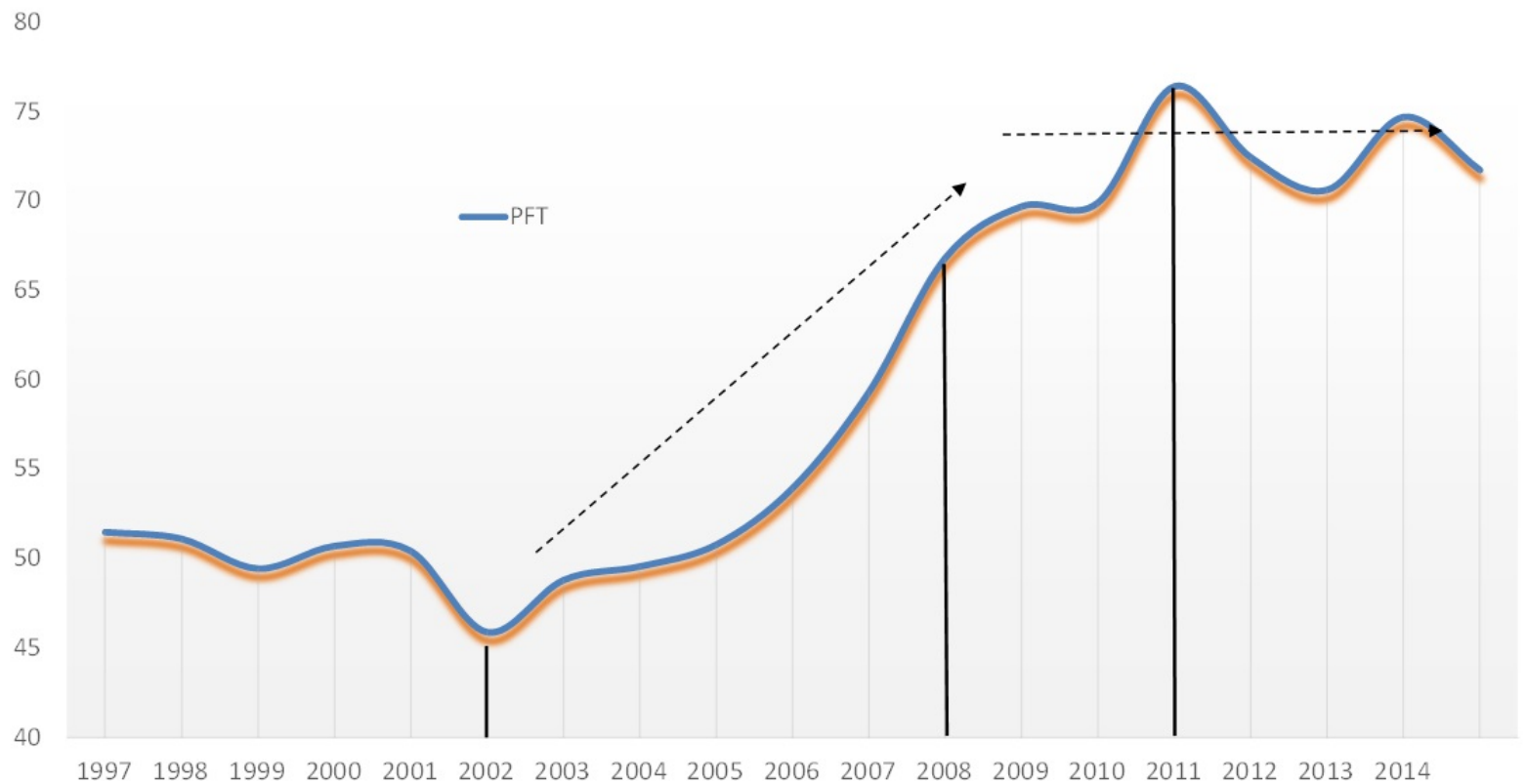

Fuente: Elaboración propia sobre la base de datos de Ministerio de Hacienda y Ministerio de Trabajo, Empleo y Seguridad Social 
Cabe aquí la pregunta de a qué se debe este estancamiento de la productividad del trabajo. Centralmente, la estrategia empresarial para resolver a su favor las tensiones distributivas estuvo guiada por los incrementos de precios más que por la inversión en capital fijo, lo cual impone un límite concreto al incremento de los ingresos empresariales a través de las formas de plusvalía relativa. Por su parte, el proceso inflacionario erosionó los salarios reales y profundizó, a su vez, la tensión distributiva.

La estrategia de retener inversiones, como tercera tensión doméstica, se presenta en espejo a la evolución de los precios. La inversión como parte del producto final cayó 8,9\% entre los años 2008 y 2011, para luego, durante la última etapa, mantener niveles por debajo del $17 \%$ (ver Figura 5). Sin embargo, como hemos mencionado, la caída de la inversión entre 2013 y 2015 no desencadenó una caída de las ganancias, debido a que se encontraron estimuladas por la redistribución regresiva de ingresos.

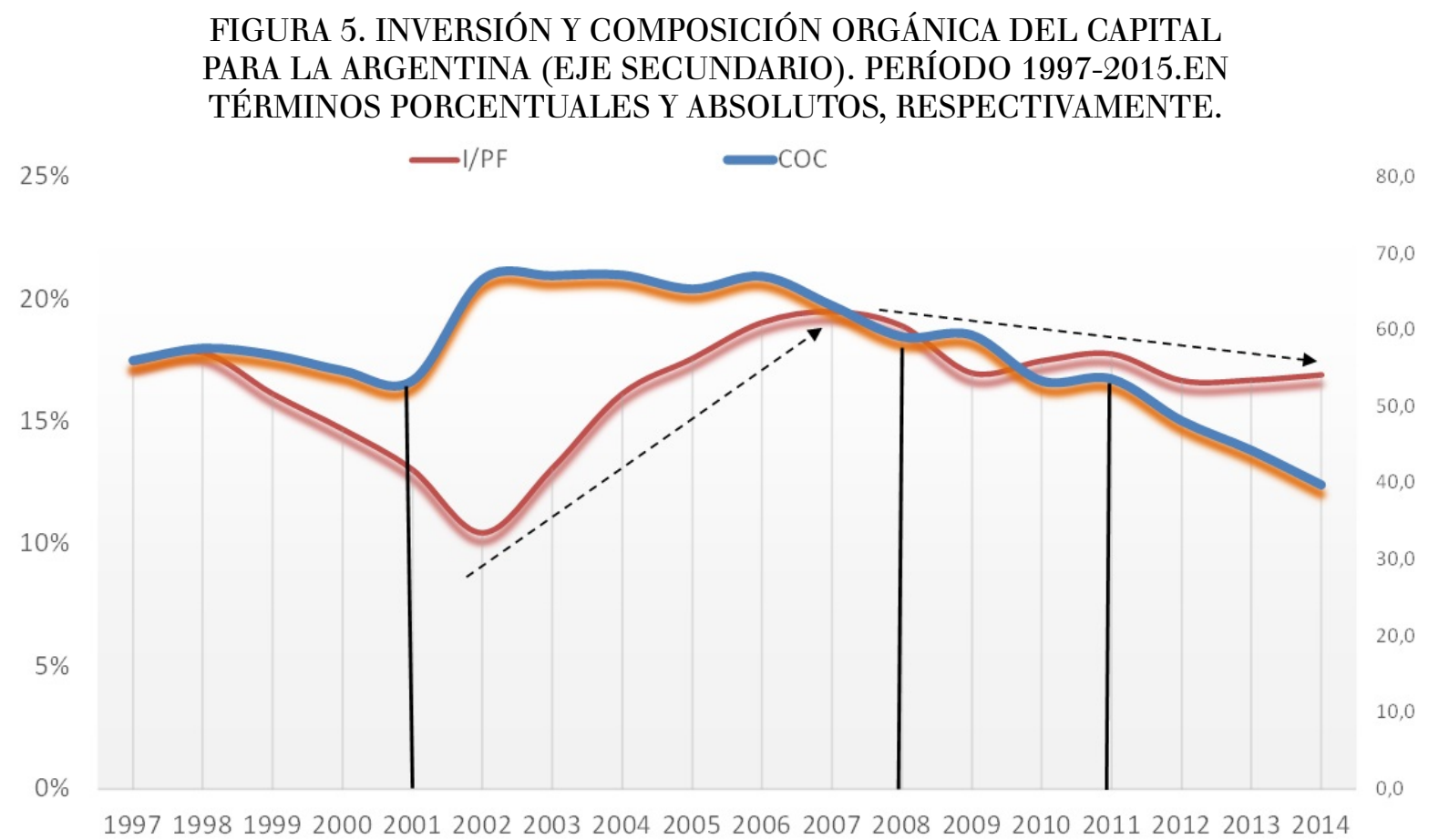

Fuente: Elaboración propia sobre la base de datos del Ministerio de Hacienda

En cuarto lugar, una de las características más estudiadas de la economía argentina para pensar los ciclos de stop and go es la estructura productiva desequilibrada (Diamand, 1972). Esto se relaciona con los diferenciales de productividad, competitividad y rentabilidad de los sectores primarios en relación con los manufactureros, lo cual da lugar a procesos de valorización con resultados muy desiguales entre sectores o ramas. En el período que estudiamos, mientras que el sector primario fue altamente rentable y competitivo internacionalmente, los sectores manufactureros expresaron registros en torno al promedio (ver Figura 6). 
FIGURA 6

Evolución del tipo de cambio real y costos unitarios laborales por rama de actividad para la Argentina. Período 1997-2015. Índice $1997=100$

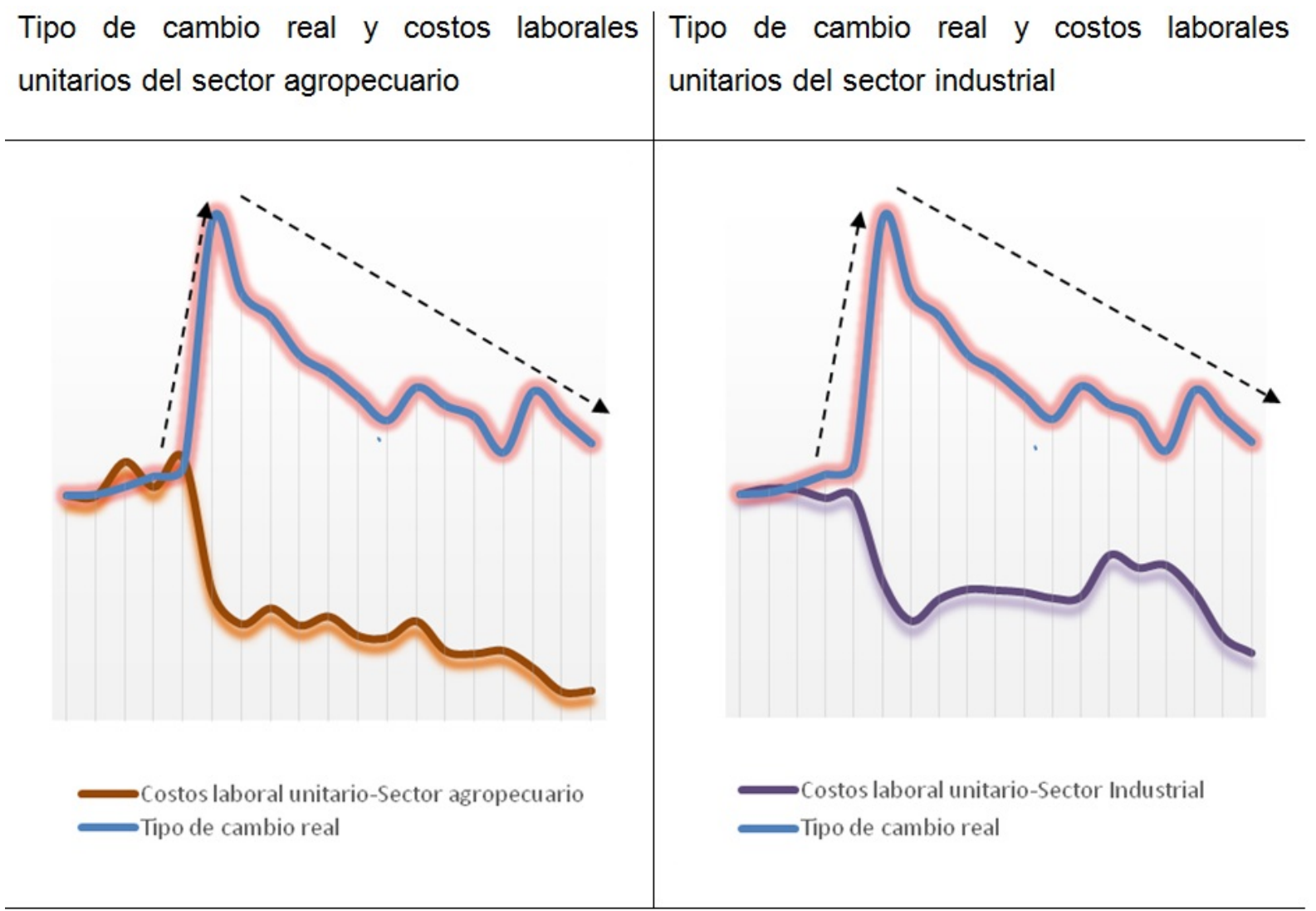

Fuente: Elaboración propia sobre la base de datos de Ministerio de Hacienda y BCRA.

De acuerdo con nuestra perspectiva, dicha estructura productiva desequilibrada tuvo dos consecuencias en el período estudiado. Por un lado, las diferencias en la rentabilidad y la capacidad competitiva de cada sector limitaron el proceso de acumulación al basarse fundamentalmente en la exportación: la capacidad exportadora de bienes primarios estuvo asociada a las variaciones en los precios internacionales que, en una economía mundial cada vez más financiarizada, tuvieron una trayectoria más inestable y estuvieron sujetos a una mayor especulación que la de bienes manufacturados. Esta dinámica también es propia de estas producciones que dependen de las variables climáticas. En este sentido, la industrialización por sustitución de importaciones fue limitada entre 2002 y 2015, debido a las tensiones inter-sectoriales y la falta de una política industrial integral (López, 2015).

Los límites competitivos del sector industrial dieron lugar a una reducida sustitución de importaciones que, entre otras variables, redundó en la reaparición de la tan mentada restricción externa (Gaggero, Schorr y Wainer, 2014). Por otro lado, las dinámicas diferenciales de producción y realización del valor en los diferentes sectores llevan a un proceso de desarrollo desigual entre las diferentes clases sociales, particularmente al interior de la clase trabajadora: aquellos grupos de trabajadores vinculados a las ramas rentables poseen, por lo general, un poder estructural mayor que aquellos que se encuentran insertos en ramas menos competitivas (Barrera Insua y López, 2016).

Finalmente, como quinto y último punto vinculado a lo anterior, la reducción del superávit comercial es la tensión externa más notoria que contribuyó al proceso de estancamiento entre 2008 y 2011. Como vemos en la tabla 2, después de 2008, la ratio de exportaciones sobre el producto final cayó desde el 19,5\% hasta registros apenas del 11,6\% en 2015 y las exportaciones netas se volvieron negativas (dando cuenta del déficit 
comercial). Esta caída del porcentaje de exportaciones sobre el producto operó como restricción de divisas en la forma más clásica de un ciclo de stop and go. En nuestra perspectiva, esta tensión en el sector externo provoca un problema de realización que refuerza las tensiones estructurales y productivas previamente descritas y, por tanto, representó uno de los aspectos más críticos del proceso de estancamiento económico del último tramo del modo de desarrollo post-neoliberal.

\section{REflexiones finales}

El presente trabajo parte de entender que las cuentas nacionales sobre las que se trabaja usualmente para el análisis de la situación económica y social de un país no son una elección anodina, siempre que se quiera desarrollar una perspectiva crítica. En ese sentido, y como parte de una línea de trabajo que hemos venido sosteniendo desde hace algunos años, comenzamos problematizando este punto y proponiendo los principales indicadores que a nuestro entender permiten comprender el proceso de acumulación y valorización del capital en la Argentina reciente.

El periodo analizado permite dar cuenta de las dimensiones económicas (esto es un patrón de reproducción del capital) que ha permitido la emergencia de una nueva configuración del modo de desarrollo a posteriori de la crisis del proyecto neoliberal en el comienzo del siglo XXI. Dicho proceso tuvo una primera etapa de valorización exitosa y alto crecimiento (2002-2007), una segunda donde emergen las tensiones y el crecimiento de la producción presenta altibajos (2008-2011), y una tercera donde las tensiones se agudizan y sobreviene el estancamiento (2012-2015).

Desde el punto de vista propuesto, dicha dinámica exhibe la profundidad de las raíces de la dependencia, las que configuran una serie de determinantes estructurales del ciclo de valorización del capital en el país. Los indicadores y series temporales construidas a partir de la reinterpretación de la Matriz Insumo Producto y sobre la base de las categorías marxianas permitieron vincular las características de la dependencia en cada momento del ciclo del capital con el éxito o fracaso del proceso de valorización.

\section{ReFERENCIAS}

Barrera, F. (2012). Informalidad $y$ valorización del capital en la Argentina reciente. Un estudio sobre su funcionalidad basado en la Matriz Insumo-Producto. [Tesis de Maestría]. Facultad Latinoamericana de Ciencias Sociales. Disponible en https://www.researchgate.net/publication/263077725_Informalidad_y_valorizacion_del_capital_en_la_ Argentina_reciente_Un_estudio_sobre_su_funcionalidad_basado_en_la_Matriz_Insumo-Producto_Informa lity_and_valorization_of_capital_in_the_recent_Argentina_A

Barrera, F. y López, E. (2010). Estimación de las categorías marxianas mediante tablas deinsumo-producto. Un análisis comparativo de Argentina y Estados Unidos. Problemas del Desarrollo. Revista Latinoamericana de Economia, 41(162), 57-83.

Barrera Insua, F. (2015). La acción sindical en el conflicto salarial de la Argentina post-convertibilidad (2006-2010). Sociedad y economía, 28, 115-136.

Barrera Insua, F. y López, E. (2016). Desigualdad salarial entre sectores económicos de la Argentina post-neoliberal: Una explicación a través de sus límites de variación. Revista de Economia Crítica, 21, 21-42.

Barrera-Insua, F. y López, E. (2019). La tasa de ganancia sectorial en la Argentina en los años posneoliberales. Realidad económica, 48(321), 35-64.

Braun, O. y Joy, L. (1981). Un modelo de estancamiento económico-Estudio de caso sobre la economía argentina. Desarrollo económico, 20(80), 585-604.

Cockshott, P., Cottrell, A. y Michaelson, G. (1995). Testing Marx: Some new resultfrom UK data. Capital \& Class, 19(1), 103-130. 
Gigliani, G. y Michelena, G. (2013). Los problemas estructurales de la industrialización en la Argentina. Realidad Económica, 278, 55-74.

Lebowitz, M. A. (2005). Más allá de "El Capital". La economía politica de la clase obrera en Marx. Madrid: Akal

López, E. (2015). Los años post-neoliberales. De la crisis a la consolidación de un nuevo modo de desarrollo. Ciudad Autónoma de Buenos Aires: Miño y Dávila Editores.

López, E. y Barrera Insua, F. (2019). The Specific Conditions of the Valorization of Capital in a Dependent Nation: The Case of Argentina (2002-2014). Review of Radical Political Economics, 51(1), 75-94.

Maniatis, T. (2005). Marxian macroeconomic categories in the Greek economy. Review of Radical Political Economics, $37(4), 494-516$.

Marini, R. M. (2007). Dialéctica de la dependencia. En C. E. Martins (Ed.), América Latina, dependenciay globalización (pp. 107-164). Buenos Aires: CLACSO-Prometeo.

Marx, K. (2006a). El Capital, Tomo 3 (Vol. 7). Mexico: Siglo XXI. Primera edición.

Marx, K. (2006b). El Capital, Tomo I ( Vol. 2). Buenos Aires: Siglo XXI Editores. Primera edición.

Marx, K. (2007). Elementos fundamentales para la crítica de la economía política: Borrador 1857-1858 (Vol. 1). México: Siglo XXI.

Memis, E. (2007). A disaggregate analysis of profit rates in Turkish manufacturing. Review of Radical Political Economics, 39(3), 398-406.

Moseley, F. (1997). The rate of profit and the future of capitalism. Review of Radical Political Economics, 29(4), 23-41.

Osorio, J. (2004). Critica de la economía vulgar: Reproducción del capital y dependencia. México: Miguel Ángel Porrúa.

Paitaridis, D. y Tsoulfidis, L. (2012). The growth of unproductive activities, the rate of profit, and the phase-change of the US economy. Review of Radical Political Economics, 44(2), 213-233.

Saad-Filho, A. (1993). A Note on Marx's Analysis of the Composition of Capital. Capital \& Class, 17(2), 127-146.

Shaikh, A. \& Tonak, E. A. (1994). Measuring the Wealth of Nations. Cambridge: Cambridge University Press.

Venida, V.S. (2007). Marxian categories empirically estimated: The Philippines, 1961-1994. Review of Radical Political Economics, 39(1), 58-79.

Wolf, E. (2001). The Recent Race of Profits the United Status. Review of Radical Political Economics, 33(3), 315-324.

\section{Notas}

1 La metodología de actualización responde a lo propuesto por MECON (2007) y redunda en una matriz con los consumos intermedios de la Argentina para precios relativos de 2004.

2 La discusión de las categorías marxianas vinculadas con trabajo productivo e improductivo que se encuentra por detrás de la reinterpretación de las cuentas nacionales ortodoxas puede verse con mayor detalle en López y Barrera Insua (2019) y Barrera y López (2010).

3 Para profundizar aspectos metodológicos sobre la reconciliación de los registros de cuentas nacionales con las categorías marxianas, puede verse Barrera y López (2010) y López y Barrera Insua (2019).

4 Para precisiones adicionales acerca de la construcción de las variables trabajadas, puede consultarse Barrera Insua y López, 2019.

5 Este fenómeno fue estudiado para la economía argentina de fines del siglo XX y principios del XXI, y se encontró que las técnicas productivas que incrementan la explotación de la fuerza de trabajo en términos absolutos, son una posible estrategia de supervivencia de los capitales subordinados (pequeñas y medianas empresas), lo que provee una explicación sistémica de los valores de informalidad estructuralmente elevados que posee el país (Barrera, 2012).

6 Dicho fenómeno fue leído por algunos analistas como el resultado de la situación de "desacople" o "desconexión” de la región respecto de las dinámicas globales que diseminaban la crisis financiera global.

\section{BY-NC-SA}

